\title{
Avaliação do conhecimento dos professores de educação física para reagirem a situações de emergência
}

\section{Assessment of physical education teachers' knowledge to react on emer- gency situations}

\author{
Dulce Esteves ${ }^{1 *}$, Paulo Pinheiro², Rui Brás ${ }^{1}$, Kelly O’Hara1 ${ }^{1}$, Ricardo Rodrigues² \\ ARTIGO ORIGINAL | ORIGINAL ARTICLE
}

\begin{abstract}
O conhecimento dos Professores de Educação Física (EF) sobre $1^{\text {os }}$ socorros condiciona a sua atuação em situações de emergência, pelo que é importante avaliá-lo. Neste sentido, desenhou-se um questionário para avaliar (1) o conhecimento teórico sobre como atuar em situações de emergência (KT); (2) o conhecimento operacional $(\mathrm{KO})$ de atuação face à emergência e (3) de que modo idade, género, habilitações literárias, perceção do conhecimento e fontes de informação influenciam KT e KO. Participaram no estudo 284 professores $(57.7 \% \mathrm{H} ; 41.9 \% \mathrm{M} ; 37.6 \pm 7.1$ anos), onde $19 \%$ dos professores entrevistados têm bom nível de KT e 49,5\% bom nível de KO. A idade e habilitações literárias influenciam o conhecimento: os mais jovens mostram melhor KT e KO e os Mestres melhor KT mas um nível igual de KO. O género não influencia nem KT nem KO. Médicos/enfermeiros e formação profissional são as melhores fontes de informação para melhorar KO. Uma vez que $50.5 \%$ dos professores apresentam nível mau ou médio de $\mathrm{KO}$, a introdução de módulos de $1^{\text {os }}$ socorros na formação profissional, especialmente se estes forem lecionados por médicos/enfermeiros, pode ser uma estratégia eficiente para melhorar o conhecimento de atuação face à emergência.
\end{abstract}

Palavras-chave: Conhecimento de $1^{\text {os }}$ socorros, acidentes escolares, segurança escolar, formação de professores

ABSTRACT

Physical Education (PE Teachers' knowledge on $1^{\text {st }}$ aid embraces their action in emergency situations, so it is important to evaluate it. We investigated 284 PE teachers (57.7\% Male, $41.9 \%$ Female, $37.6 \pm$ 7.1 years) using a questionnaire specially designed to evaluate (1) theoretical knowledge (KT) about how to act in emergency situations, (2) operational knowledge (KO) for action in the face of emergency and (3) how age, gender, education, perception of individual knowledge and information sources of influence KT and KO. 19\% of interviewed teachers present good level of KT and $49.5 \%$ good level of KO. Age and education influence knowledge: younger teachers show best KT and KO Masters present better KT but an equal level of KO. Gender does not influence neither KT nor KO. Doctors / nurses and professional education are the best information sources to improve KO. 50.5\% of teachers have a bad or average $\mathrm{KO}$, which may impair their action face the accident. The introduction of modules in $1^{\text {st }}$ aid training, especially if they are taught by doctors / nurses, seems to be an efficient strategy to improve the knowledge of action on emergency.

Keywords: $1^{\text {st }}$ aid knowledge, school accidents, school safety, teachers' education

Artigo recebido a 27.10.2013; Aceite 22.05.2014

${ }^{1}$ Departamento de Ciências do Desporto, Universidade da Beira Interior, Covilhã, Portugal

2 Departamento de gestão, Universidade Beira Interior, Covilhã, Portugal

* Autor correspondente: Departamento de Ciências do Desporto, Universidade da Beira Interior 6200-001 Covilhã; E-mail: desteves@ubi.pt 


\section{INTRODUÇÃO}

Os benefícios da atividade física e desportiva estão bem documentados (por exemplo Bouchard, Blair, \& Haskell, 2012) e quer as aulas de Educação Física como o Desporto Escolar são considerados elementos chave para a promoção de uma vida ativa nas crianças e jovens (Strong et al., 2005; World Health Organization, 2010). No entanto, as atividades físicas e desportivas são a maior causa de lesões e acidentes nos adolescentes e jovens (idades 11-18 anos) quer na Europa quer na América do Norte (Abernethy, MacAuley, McNally, \& McCann, 2003; Başer, Coban, Taşci, Sungur, \& Bayat, 2007; Orchard \& Finch, 2002; K.-M. Wang, Lin, \& Huang, 2012). Prédine et al. (2002) realizaram um estudo epidemiológico baseado em questionários aplicados a 2.396 adolescentes que sofreram acidentes escolares, e que recorreram à enfermaria da escola, constatando que $52,8 \%$ dos acidentes ocorriam durante as atividades desportivas, enquanto apenas $12,7 \%$ ocorriam nas atividades de recreação. Linakis, Amanullah e Mello (2006) referem que em cada ano escolar 3,7 milhões de crianças norte-americanas (5-19 anos) sofrem ferimentos graves na escola, o que representa $16,5 \%$ de todas as visitas anuais às urgências hospitalares. A literatura refere que cerca de um quarto destes acidentes resultam em situações graves, como fraturas ou deslocamentos ósseos, rutura de ligamentos, lesões da coluna ou cerebrais, enquanto os restantes acidentes provocam danos menores, como entorses, distensões, contusões, escoriações, e lacerações (Abernethy et al., 2003; Abraldes \& Ortín, 2010; Emery \& Tyreman, 2009). Num estudo realizado em Portugal, Reis (2005) concluiu que dos 2625 acidentes escolares analisados, $56.0 \%$ foram quedas, $24.5 \%$ choques, $2.6 \%$ manipulações de objetos, e $15.7 \%$ outros acidentes.

Apesar dos acidentes fatais serem muito raros Miller e Spicer (1998), num relatório sobre acidentes relacionados com o desporto na escola, identificaram 30 mortes na faixa etária de 15-19 anos, num período de 6 anos.
Os benefícios da prática desportiva tanto na saúde mental como física de adolescentes e jovens é inquestionável (Strong et al., 2005), mas apesar da sua importância, a ausência de conhecimento adequado e da capacidade para lidar com situações de emergência provenientes de acidentes pode diminuir significativamente as vantagens dessa prática, podendo por em risco a saúde dos estudantes (K.-M. Wang et al., 2012). As aulas de Educação Física e o Desporto Escolar têm de ser o mais seguras possível, se se pretendem implementar estratégias que levem os alunos a gostarem da atividade física a adotarem um estilo de vida ativo.

O professor de Educação Física é aquele que tem maior probabilidade de assistir a um acidente escolar, logo é frequentemente o primeiro agente responsável por prestar cuidados após um acidente. Vários estudos (Abernethy et al., 2003; Abraldes \& Ortín, 2010; Junkins et al., 1999) indicam que a percentagem de lesões que ocorrem nas aulas de Educação Física varia entre $17,5 \%$ e $23 \%$ em relação ao total de acidentes escolares. Assim, o professor de Educação Física tem um papel fundamental na prestação de cuidados aos alunos (Fioruc, Molina, Junior, \& Lima, 2008; Liberal, Aires, Aires, \& Osório, 2005), devendo estar preparado para agir de maneira eficiente, segura e adequada (Abraldes \& Ortín, 2010; Patsaki et al., 2012), uma vez que a sua ação tem consequências na recuperação dos alunos (Başer et al., 2007; Flegel, 2002; Olympia, Wan, \& Avner, 2005). Na literatura encontram-se várias investigações (Başer et al., 2007; Liberal et al., 2005; Olympia et al., 2005; K.-M. Wang et al., 2012) com resultados consensuais sobre a necessidade do professor de Educação Física estar preparado para reagir em situações de emergência.

Sendo assim, o professor de Educação Física tem de ter competências específicas que lhe permitam lidar convenientemente com os acidentes. Para isso podem necessitar de uma formação específica ao nível de $1^{\text {os }}$ socorros, o que nem sempre acontece. Flegel (2002) refere que 
nos Estados Unidos apenas metade dos docentes de Educação Física têm curso de primeiros socorros.

Então a questão que se coloca é se de fato os docentes estão preparados para reagir aos acidentes que potencialmente possam acontecer.

Alguma literatura refere que os professores estão mal preparados para lidar com os possíveis acidentes, pois o seu conhecimento é insuficiente ou não possuem o discernimento e a capacidade para atuarem caso seja necessário (Emery \& Tyreman, 2009; Ransone \& DunnBennett, 1999; K.-M. Wang et al., 2012). Vários autores apontam para o insuficiente conhecimento que os professores têm sobre $1^{\text {os }}$ socorros como a principal causa da incapacidade de lidar com situações de emergência (Abernethy et al., 2003; Başer et al., 2007; Fioruc et al., 2008; K.M. Wang et al., 2012).

Por outro lado, os estudos de Patsaki et al. (2012) e Abraldes e Ortín (2010) concluem que os professores têm um bom conhecimento de $1^{\text {os }}$ socorros e boa capacidade de lidar com situações de emergência.

Uma vez que: 1) não existe consenso na literatura sobre se os professores têm ou não competência para agir nestas situações, 2) o conhecimento de $1^{\text {os }}$ socorros é visto como uma condição essencial para se ter uma boa prestação perante acidentes/situações de emergência e 3) o estudo do conhecimento sobre $1^{\text {os }}$ socorros dos professores de EF portugueses são escassos, o presente trabalho tem como objetivo geral avaliar se os professores de Educação Física portugueses têm conhecimento adequado para atuar em situações de emergência. Além deste objetivo principal, pretendemos identificar se fatores como idade, género, habilitações literárias/formação académica, perceção do conhecimento ou fontes de informação sobre conhecimento de $1^{\text {os }}$ socorros, influenciam esse conhecimento.

\section{MÉTODO}

Este estudo pertence a uma investigação de tipo transversal analítico em que a variável dependente é o conhecimento dos professores de educação física para agirem em situações de emergência e as variáveis independentes são a idade, o género, as habilitações literárias, a perceção do conhecimento e as fontes de informação sobre $1^{\text {os }}$ socorros.

\section{Amostra}

A amostra foi constituída por professores de Educação Física de Portugal que estão no ensino há pelo menos 3 anos (critério de seleção). Foi utilizado o método de seleção não aleatória tendo-se obtido uma amostra de 279 professores, que representa $4.4 \%$ total dos professores de Educação Física de Portugal, distribuídos pelo território nacional (continente e ilhas).

A amostra foi constituída por $57.7 \%$ homens e $41.9 \%$ mulheres, com uma média de idades de $37.6 \pm 7.1$ anos. Relativamente às habilitações literárias $67.9 \%$ são licenciados, $28.8 \%$ mestres e apenas $1.7 \%$ têm doutoramento. Quanto ao nível de ensino, $13.4 \%$ lecionam no $1^{\circ}$ ciclo, $30.9 \%$ no $2^{\circ}$ ciclo, $24.3 \%$ no $3^{\circ}$ ciclo e $29.6 \%$ no ensino secundário. Relativamente aos anos de docência de cada elemento questionado, $44 \%$ encontram-se entre os 9 a 14 anos de docência, seguidos por $27.1 \%$ com 3 a 8 anos de atividade docente.

\section{Instrumentos}

Foi aplicado um questionário aos professores de Educação Física com mais de 3 anos de docência com objetivo de avaliar o conhecimento para reagirem a situações de emergência durante as aulas. O questionário foi construído para avaliar: (1) o conhecimento teórico (KT) dos professores sobre como agir face a situações de emergência - grupos de questões O1; (2) o conhecimento operacional (KO) perante essas situações de emergência- grupo de questões $\mathrm{O}$; (3) a perceção que os professores têm do seu próprio conhecimento - grupo de questões O3; e (4) as principais fontes de informação sobre $1^{\circ} \mathrm{S}$ socorros usadas pelos professores - grupo de questões $\mathrm{O} 4$. O questionário teve uma validação de conteúdos feita por especialistas. 
O grupo de questões $\mathrm{O} 1$ para avaliar o conhecimento teórico (KT) dos docentes foi constituído por perguntas de carácter mais teórico, como conceitos, identificações de diferentes tipos de lesões e tratamentos indicados para determinadas situações. O grupo de questões O2 para avaliar conhecimento operacional (KO) dos docentes foi constituído por perguntas mais operativas, nomeadamente ao nível da tomada de decisão. As questões dos grupos $\mathrm{O} 1$ e $\mathrm{O} 2$ foram extraídas de vários estudos científicos (Abernethy et al., 2003; Abraldes \& Ortín, 2010; Ransone \& Dunn-Bennett, 1999; C. K. J. Wang \& Koh, 2006) e estavam estruturadas sob a forma de escolha múltipla.

Para uma melhor compreensão da pontuação obtida nos grupos $\mathrm{O} 1$ e $\mathrm{O} 2$ do questionário, criámos uma variável qualitativa, segundo a percentagem de respostas certas obtidas. Assim, para ambos os grupos foi dada uma classificação ("Mau", "Médio" e "Bom"), consoante a \% de acerto a cada uma das questões: "Mau" se o professor respondeu corretamente a menos ou a $50 \%$ das questões, "Médio" se respondeu corretamente a 50-80\% das questões e "Bom" se respondeu a mais de $80 \%$ das questões. A escolha das diferentes percentagens correspondentes às classificações foi realizada de modo semelhante à metodologia apresentada por Abraldes e Ortin (2010), que consideram "mau" ou "muito mau" percentagens de resposta abaixo dos $50 \%$.

O grupo de questões $\mathrm{O} 3$ foi estabelecido, especificamente para esta investigação para avaliar a perceção que os professores têm do seu próprio conhecimento. Neste grupo as respostas foram dadas numa escala de Likert de concordância ( $1=$ Discordo completamente; $5=$ Totalmente de acordo).

Do conjunto de questões $\mathrm{O} 3$ consideramos que a perceção geral do conhecimento sobre $1^{\text {os }}$ socorros é avaliada fundamentalmente pelas questões O3.2 "Estou bem informado sobre o que fazer em caso de acidente" e O3.3 "Tenho bastantes dúvidas sobre como aplicar primeiros socorros, dado que as restantes questões avaliam aspetos particulares de emergência, pelo que foram estas questões analisada.
O grupo de questões $\mathrm{O} 4$ foi adaptado de $\mathrm{Pi}$ nheiro, Esteves e Brás (2011) relativamente às principais fontes de informação usadas em Exercício e Saúde, tendo também sido usado uma escala de Likert de concordância ( $1=$ Discordo completamente; $5=$ Totalmente de acordo).

\section{Procedimentos}

A recolha de dados da presente investigação ocorreu entre Janeiro e Abril de 2013 e fez-se simultaneamente por via eletrónica (nível nacional e ilhas) e em formato de papel (nível regional - os professores que responderam em formato papel eram alertados para não responderem ao mesmo questionário por via eletrónica), de modo a poder abranger o máximo de dispersão geográfica. Como meio de divulgação o estudo teve a colaboração dos sindicatos nacionais e dos serviços administrativos de diferentes associações de professores.

\section{Análise Estatística}

Os dados foram tratados no programa Excel Office 2007 e IBM SPSS Statistics 19. Fez-se uma análise estatística descritiva básica (médias, desvios padrão, percentagens, valores máximos e mínimos). A análise inferencial das variáveis fez-se após de verificar a normalidade (teste de Kolmogorov-Smirnov) e a homogeneidade das mesmas (teste de Levene).

Para as questões O3, foram determinados os valores de Cronbach Alpha para avaliar a sua validade e a fiabilidade

De modo a comparar estatisticamente os diferentes níveis de conhecimento ("Mau", "Médio", "Bom") relativamente a KT e KO e relacioná-los com os diferentes parâmetros em análise, fez-se uma análise da variância (Anova), aplicando o post hoc Tukey B ou, em caso das variáveis apresentarem valores discretos, um teste de Qui-quadrado. Em todas as análises, a significância estatística foi aceite para um valor de $95 \%(p<0.05)$. 


\section{RESULTADOS}

Os resultados obtidos na presente investigação mostram que a grande maioria dos professores entrevistados têm um nível médio de conhecimentos sobre o que fazer em situações de emergência, visto que a classificação de "Mau" não abrange uma porção considerável dos docentes (Tabela 1).

Tabela 1

Nível de conhecimento para atuar em situações de emergência

\begin{tabular}{cccc}
\hline Tipo de Conhecimento & Mau $(<\mathbf{5 0 \%}$ acerto) & Médio $(\mathbf{5 0 - 8 0 \%}$ acerto) & Bom (> 80\% acerto) \\
\hline Teórico (KT) & $22.9 \%$ & $58.1 \%$ & $19.0 \%$ \\
Operativo (KO) & $9.0 \%$ & $41.6 \%$ & $49.5 \%$ \\
\hline
\end{tabular}

Os resultados obtidos mostram que a percentagem de $\mathrm{KO}$ é bastante superior ao KT, no entanto há que realçar que mais de metade dos professores entrevistados não tem um nível "Bom " de conhecimento operacional.

\section{Fatores que podem influenciar o nível de co- nhecimento teórico e operacional}

Interessa agora avaliar quais os fatores que afetam o nível de conhecimento teórico (KT) e o nível de conhecimento operacional (KO), mais especificamente, consideramos o efeito da a) idade, b) habilitações literárias, c) perceção do próprio conhecimento e d) fontes de informação no nível de KT e KO.

\section{Idade}

Quanto à idade, consideramos 4 grupos etários (Grupo 1: < 30 anos; Grupo 2: 30-40 anos; Grupo 3: 41-50 anos; Grupo 4: > 50 anos), e fez-se uma análise descritiva (Tabela 2) sobre a $\%$ de classificações de "Bom/Médio/Mau" de $\mathrm{KT}$ e KO, para os diferentes grupos investigados.

Tabela 2

Distribuição das respostas "Mau/Médio/Bom" para KT e KO, segundo a idade

\begin{tabular}{ccccccc}
\hline \multicolumn{3}{c}{ KT } & \multicolumn{3}{c}{ KO } \\
\hline $\begin{array}{c}\text { Idade } \\
\text { (anos) }\end{array}$ & \% Mau & \% Médio & \% Bom & \% Mau & \% Médio & \% Bom \\
\hline$<30$ & 20.5 & 43.6 & 35.9 & 2.6 & 41.0 & 56.4 \\
$30-40$ & 22.4 & 62.7 & 14.9 & 8.7 & 46.6 & 44.7 \\
$41-50$ & 20.0 & 60.0 & 20.0 & 7.1 & 35.7 & 57.1 \\
$>50$ & 42.9 & 42.9 & 14.3 & 35.7 & 21.4 & 42.9 \\
\hline
\end{tabular}

De seguida, fez-se um teste de comparação entre os grupos (ANOVA), e um teste Post Hoc (Tukey B). Os resultados, descritos na Tabela 3, mostram que existem diferenças significativas entre os grupos etários investigados (para $p<$ $0.05)$, quer para o KT quer para o KO. Verificamos que os professores mais jovens $(<30$ anos) apresentam estatisticamente um melhor KT relativamente ao mais velhos ( $>50$ anos), enquanto que estes apresentam um KO significativamente mais baixo que os docentes mais novos. 
Tabela 3

Variação do KT e $K O$, segundo a idade

\begin{tabular}{ccccc}
\hline \multirow{2}{*}{ Tukey B } & \multicolumn{2}{c}{ KT } & \multicolumn{2}{c}{ KO } \\
& \multicolumn{2}{c}{ Subconjunto para alpha=.05 } & G2 & Subconjunto para alpha $=.05$ \\
\hline Idade (anos) & G1 & & G1 & G2 \\
\hline$<30$ & 2.15 & 1.93 & 2.36 & 2.54 \\
$30-40$ & 1.93 & 2.00 & & 2.50 \\
$41-50$ & 2.00 & 1.71 & 2.07 & \\
$>50$ & & & & \\
\hline
\end{tabular}

Género

Como já referimos, a amostra dos professores entrevistados é constituída por $57.7 \%$ ho- mens e $41.9 \%$ mulheres. A distribuição das classificações "Bom/Médio/Mau" de KT e KO, segundo o género, estão descritas na Tabela 4.

Tabela 4

Distribuição das respostas "Mau/Médio/Bom" para KT e KO, segundo o género

\begin{tabular}{ccccccc}
\hline & \multicolumn{3}{c}{ KT } & \multicolumn{3}{c}{ KO } \\
\hline Género & \% Mau & \% Médio & \% Bom & \% Mau & \% Médio & \% Bom \\
\hline Feminino & 26.1 & 58.8 & 15.1 & 10.1 & 38.7 & 51.2 \\
Masculino & 20.1 & 27.9 & 21.6 & 7.3 & 44.5 & 48.2 \\
\hline
\end{tabular}

Pela análise descritiva, nota-se que não há grandes diferenças na distribuição das classificações, entre géneros. De modo a verificar se o género, enquanto variável biológica, influencia o KT e o KO, procedemos ao teste do Qui-quadrado $\left(x^{2}\right)$. Os resultados mostram que o género não influencia nem o KT $(p=0.251)$ nem o KO $(p=0.517)$, para uma significância estatística de $95 \%$.

Tabela 5

Distribuição das respostas "Mau/Médio/Bom" para KT e KO, segundo as habilitaçôes literárias

\begin{tabular}{ccccccc}
\hline & \multicolumn{3}{c}{ KT } & \multicolumn{3}{c}{ KO } \\
\hline $\begin{array}{c}\text { Habilitações } \\
\text { literárias }\end{array}$ & \% Mau & \% Médio & \% Bom & \% Mau & \% Médio & \% Bom \\
\hline Licenciatura & 18.3 & 36.9 & 13.6 & 8.2 & 28.7 & 31.9 \\
Mestrado & 3.6 & 20.8 & 5.0 & .72 & 11.8 & 16.8 \\
Doutoramento & 1.1 & .36 & .36 & 0 & 1.1 & .72 \\
\hline
\end{tabular}

Na Tabela 5 observamos que a \% de "Mau" é bastante superior nos licenciados, face aos

\section{Habilitações literárias}

Relativamente às habilitações literárias a amostra avaliada é constituída por $67,9 \%$ licenciados, $28,8 \%$ mestres e $1,7 \%$ doutorados. A distribuição dos níveis de conhecimento em estudo (KO e KT) está descrita na Tabela 5, para as diferentes habilitações literárias. mestres. De modo a compreender se a influência das habilitações literárias em cada um dos 
conhecimentos investigados é estatisticamente significativa, procedeu-se ao teste do Qui-quadrado $\left(x^{2}\right)$. Os resultados estatísticos provenientes deste teste não contemplam os doutorados, dado este ser um número residual na amostra, sendo excluídos (contagem esperada $<5$ ).

Para o KT verifica-se que existem diferenças significativas $(p=0.013)$ entre os 2 grupos em análise (licenciados e mestrados). O nível de habilitações literárias influencia o KT, sendo que os mestres têm um maior nível de KT que os licenciados. Para KO, não existem diferenças estatísticas entre os dois grupos em análise, para um nível de significância de $95 \%(p=0.084)$.

Os resultados mostram que as habilitações literárias influenciam o KT sobre os $1^{\text {os }}$ socorros, uma vez que os professores com mestrado apresentam melhores níveis deste conhecimento, mas não têm uma grande influência no $\mathrm{KO}$, no modo como o professor atua, face a situações de emergência. Isto significa que o mestrado não melhora as competências que o professor tem, para lidar com acidentes/emergências.

\section{Perceção do próprio conhecimento}

A matriz de correlações (apresentada na Tabela 6) indica que as diversas questões $\mathrm{O} 3$ estão correlacionadas, pelo que estas podem fazer parte do mesmo grupo de questões. O Alpha de Cronbach para o conjunto de questões $\mathrm{O} 3$ tem um valor de 0.812 , o que indicou um elevado nível de fiabilidade.

Tabela 6

Matriz de correlaçôes dos itens de perceção de conhecimento

\begin{tabular}{|c|c|c|c|c|c|c|c|c|c|c|c|c|}
\hline & O3.1 & 03.2 & O3.3 & O3.4 & 03.5 & 03.6 & O3.7 & 03.8 & O3.9 & O3.10 & O3.11 & O3.12 \\
\hline O3.1 & 1.000 & & & & & & & & & & & \\
\hline O3.2 & 0.100 & 1.000 & & & & & & & & & & \\
\hline O3.3 & 0.062 & -.333 & 1.000 & & & & & & & & & \\
\hline O3.4 & 0.055 & .308 & -0.281 & 1.000 & & & & & & & & \\
\hline O3.5 & 0.155 & .409 & -0.305 & 0.616 & 1.000 & & & & & & & \\
\hline O3.6 & 0.206 & .278 & -0.222 & 0.381 & 0.417 & 1.000 & & & & & & \\
\hline 03.7 & 0.235 & .180 & -0.172 & 0.336 & 0.396 & 0.509 & 1.000 & & & & & \\
\hline 03.8 & 0.176 & 0.320 & -0.231 & 0.461 & 0.506 & 0.592 & 0.472 & 1.000 & & & & \\
\hline O3.9 & 0.156 & 0.340 & -0.238 & 0.302 & 0.416 & 0.498 & 0.368 & 0.594 & 1.000 & & & \\
\hline O3.10 & 0.083 & 0.216 & -0.302 & 0.322 & 0.321 & 0.427 & 0.320 & 0.275 & 0.412 & 1.000 & & \\
\hline O3.11 & 0.115 & 0.309 & -0.285 & 0.325 & 0.428 & 0.531 & 0.362 & 0.574 & 0.583 & 0.471 & 1.000 & \\
\hline O3.12 & 0.030 & 0.255 & -0.281 & 0.275 & 0.327 & 0.376 & 0.366 & 0.478 & 0.504 & 0.300 & 0.489 & 1.000 \\
\hline
\end{tabular}

O3.1 Sei quais os benefícios que os primeiros socorros trazem à minha profissão; 03.2-Estou bem informado sobre o que fazer em caso de acidente; 03.3-Tenho bastantes dúvidas sobre como aplicar primeiros socorros; O3.4 Estou preparado/a para atuar em caso de fraturas; O3.5 Estou preparado/a para atuar em caso de desmaios (perdas de consciência); O3.6 Estou preparado/a para atuar em caso de cortes/feridas profundas; O3.7 Estou preparado/a para atuar em caso de quebras de açúcar (hipoglicemias); O3.8 Estou preparado/a para atuar em caso de hemorragias; O3.9 Estou preparado/a para atuar em caso de convulsões; O3.10 Estou preparado/a para atuar em caso do aluno deixar de respirar (paragens respiratórias); O3.11 Estou preparado/a para atuar em caso do aluno ter uma lesão nos olhos (lesão ocular/oftalmológica); O3.12 Estou preparado/a para atuar em caso do aluno ter uma lesão na coluna

Ao nível da relação da perceção individual de conhecimento com o nível do conhecimento, da análise descritiva dos resultados obtivemos que $46,6 \%$ dos professores de educação física consideram estar bem informados sobre como atuar em caso de emergência (questão 03.2) e apenas 23,2\% dizem não sentir grandes dúvidas sobre como aplicar os $1^{\text {os }}$ socorros (questão 03.3). 
Relativamente à questão 03.2 "Estou bem informado sobre o que fazer em caso de acidente", os docentes que apresentam nível "Mau" de KT são estatisticamente diferentes dos docentes que apresentam nível "Médio" $(p=0.013)$ (Tabela 7), tendo uma perceção de não estarem tão bem informados sobre o que fazer em caso de acidente. No entanto não encontrámos diferenças significativas entre os docentes que apresentam nível "Mau" e nível "Bom". No que diz respeito ao KO não existem diferenças significativas entre os diferentes níveis de conhecimento.
Quanto à questão 03.3 "Tenho bastantes dúvidas sobre como aplicar primeiros socorros" (Tabela 9), os resultados mostram que existem apenas diferenças significativas entre os docentes com nível "Mau" e "Bom" de conhecimento, quer para KT $(p=0.017)$ quer para KO $(p=0.016)$, apresentado os docentes com nível "Mau" mais dúvidas sobre como aplicar os $1^{\text {os }}$ socorros.

Tabela 7

Médias do KT e KO, segundo a perceção de conhecimento, e significância das respetivas diferenças

\section{O3.2 Estou bem informado sobre o que fazer em caso de acidente \\ KT \\ O3.3. Tenho bastantes dúvidas sobre como aplicar primeiros socorros \\ KT KO}

\begin{tabular}{ccccc} 
& KT & KO & KT & KO \\
\hline Mau vs. Médio & 3.06 vs. 3.43 & 3.24 vs 3.30 & 3.14 vs 2.83 & 3.32 vs 2.94 \\
& $(p=0.013)^{*}$ & $(p=0.954)$ & $(p=0.087)$ & $(p=0.184)$ \\
Mau vs. Bom & 3.06 vs. 3.43 & 3.24 vs 3.41 & 3.14 vs 2.41 & 3.32 vs 2,73 \\
& $(p=0.064)$ & $(p=0.646)$ & $(p=0.017)^{*}$ & $(p=0.016)^{*}$ \\
Médio vs. Bom & 3.43 vs. 3.43 & 3.30 vs 3.41 & 2.83 vs 2.41 & 2.94 vs 2.73 \\
& $(p=0.999)$ & $(p=0.557)$ & $(p=0.412)$ & $(p=0.202)$ \\
\hline
\end{tabular}

3.14 vs $2.83 \quad 3.32$ vs 2.94

$(\mathrm{p}=0.087)$

\section{Fontes de informação}

Outro fator considerado na presente investigação, como podendo influenciar o nível de conhecimentos dos professores sobre como atuar em caso de emergência, foi a proveniência da informação sobre $1^{\text {os }}$ socorros, isto é, qual a origem das informações que os professores de educação física têm sobre esta matéria (Tabela 8).

Analisando a Tabela 8, observámos que as principais fontes de informação referidas pelos professores de educação física são: médicos/enfermeiros (41.4\%), formação profissional (36.2) e a licenciatura (34.4). As fontes menos utilizadas são: redes sociais (3.3\%) e amigos/família (9.7\%).

De seguida, fez-se um teste de comparação entre os grupos (ANOVA), e um teste Post Hoc (Tukey B). Os resultados estão descritos nas Tabelas 9 e 10.
No que diz respeito à comparação dos níveis KT (Tabela 9) com as diferentes fontes de informação utilizadas de um modo geral não observámos diferenças significativas entre os diferentes níveis de conhecimento. No entanto tem-se que os docentes com um nível "Bom" de KT recorrem significativamente mais a "Revistas/jornais/TV" do que os docentes com nível "Médio" $(p=0.000)$ e do que os docentes com nível "Mau" $(p=0.001)$. Além disso os docentes com um nível "Médio" de KT recorrem significativamente menos a "Colegas Profissionais de Desporto " do que os docentes com nível "Bom" $(p=0.041)$ e do que os docentes com nível "Mau" $(p=0.028)$.

Relativamente à influência das fontes de informação sobre o KO (Tabela 10), também de um modo geral não observámos diferenças significativas entre os três níveis de conhecimento. Porém os resultados mostram que os 
docentes com nível "Bom" de KO recorrem significativamente mais a "Médicos/Enfermeiros" do que os docentes com nível "Mau" $(p=0.003)$. Por outro lado os docentes com nível "Mau" de KO procuram significativamente menos a "Formação profissional" do que os docentes com nível "Bom" $(p=0.017)$ e do que os docentes com nível "Médio" $(p=0.018)$ para obtenção de informação. Verificamos também que apesar das "Redes sociais na internet" serem pouco consultadas, os docentes com nível "Bom" de KO procuram significativamente menos do que os docentes com nível "Médio" $(\mathrm{p}=0.001)$ e do que os docentes com nível "Mau" $(p=0.049)$.

Tabela 8

Principais fontes de informação sobre $1^{o s}$ socorros usadas pelos professores de Educação Física

\begin{tabular}{lccc} 
& \% de uso frequente & Média & s \\
\hline Médicos/Enfermeiros & 41.4 & 3.23 & 1.186 \\
Formação profissional & 36.2 & 3.14 & 1.013 \\
Licenciatura & 34.4 & 3.00 & 1.067 \\
Colegas profissionais de Ed. Física & 16.6 & 2.63 & 0.886 \\
Sites Internet & 14.6 & 2.53 & 0.922 \\
Revistas/Jornais/TV & 10.1 & 2.47 & 0.890 \\
Outros colegas professores & 10.8 & 2.39 & 0.902 \\
Amigos/Família & 9.7 & 2.27 & 1.032 \\
Redes Sociais na Internet & 3.3 & 1.52 & 0.857 \\
\hline
\end{tabular}

Tabela 9

Médias do KT segundo a influência da fonte de informação sobre $1^{\text {os }}$ socorros, e significância das respetivas diferenças

\begin{tabular}{|c|c|c|c|}
\hline \multirow{2}{*}{ Fontes de Informação } & \multicolumn{3}{|c|}{ KT } \\
\hline & Mau vs. Médio & Mau vs. Bom & Médio vs. Bom \\
\hline Médicos/Enfermeiros & $\begin{array}{c}3.00 \text { vs. } 3.33 \\
(p=0.150)\end{array}$ & $\begin{array}{c}3.00 \text { vs. } 3.21 \\
(p=0.609)\end{array}$ & $\begin{array}{c}3.33 \text { vs. } 3.21 \\
(p=0.806)\end{array}$ \\
\hline Revistas/Jornais/TV & $\begin{array}{c}2.32 \text { vs. } 2.36 \\
\quad(p=1.000)\end{array}$ & $\begin{array}{c}2.36 \text { vs. } 2.94 \\
(p=0.001)^{*}\end{array}$ & $\begin{array}{c}2.36 \text { vs. } 2.94 \\
(p=0.000)^{*}\end{array}$ \\
\hline Amigos/familiares & $\begin{array}{l}2.35 \text { vs. } 2.11 \\
(p=0.240)\end{array}$ & $\begin{array}{c}2.35 \text { vs. } 2.66 \\
(p=0.242)\end{array}$ & $\begin{array}{c}2.11 \text { vs. } 2.66 \\
(p=0.002)^{*}\end{array}$ \\
\hline Licenciatura & $\begin{array}{c}3.08 \text { vs. } 2.97 \\
(p=0.765)\end{array}$ & $\begin{array}{c}3.08 \text { vs. } 3.02 \\
(p=0.950)\end{array}$ & $\begin{array}{l}2.97 \text { vs. } 3.02 \\
(p=0.954)\end{array}$ \\
\hline Formação profissional & $\begin{array}{c}3.13 \text { vs. } 3.12 \\
(p=0.998)\end{array}$ & $\begin{array}{c}3.13 \text { vs. } 3.23 \\
(p=0.862)\end{array}$ & $\begin{array}{c}3.12 \text { vs. } 3.23 \\
(p=0.782)\end{array}$ \\
\hline Sites Internet & $\begin{array}{l}2.61 \text { vs. } 2.44 \\
(p=0.435)\end{array}$ & $\begin{array}{c}2.61 \text { vs. } 2.74 \\
(p=0.750)\end{array}$ & $\begin{array}{l}2.44 \text { vs. } 2.74 \\
\quad(p=0.103)\end{array}$ \\
\hline Colegas profissionais de desporto & $\begin{array}{c}2.83 \text { vs. } 2.50 \\
(p=0.028)^{*}\end{array}$ & $\begin{array}{c}2.83 \text { vs. } 2.83 \\
(p=1.000)\end{array}$ & $\begin{array}{c}2.49 \text { vs. } 2.83 \\
(p=0.041)^{*}\end{array}$ \\
\hline Colegas professores & $\begin{array}{c}2.58 \text { vs. } 2.28 \\
\quad(p=0.068)\end{array}$ & $\begin{array}{c}2.58 \text { vs. } 2.53 \\
(p=0.943)\end{array}$ & $\begin{array}{l}2.28 \text { vs. } 2.53 \\
\quad(p=0.193)\end{array}$ \\
\hline Redes sociais na internet & $\begin{array}{c}1.69 \text { vs. } 1.43 \\
(p=0.115)\end{array}$ & $\begin{array}{c}1.69 \text { vs. } 1.59 \\
(p=0.794)\end{array}$ & $\begin{array}{c}1.43 \text { vs. } 1.59 \\
(p=0.498)\end{array}$ \\
\hline
\end{tabular}


Tabela 10

Médias do KO segundo a influência da fonte de informação sobre $1^{\text {os }}$ socorros, e significância das respetivas diferenças

\begin{tabular}{|c|c|c|c|}
\hline \multirow{2}{*}{ Fontes de Informação } & \multicolumn{3}{|c|}{ KO } \\
\hline & Mau vs. Médio & Mau vs. Bom & Médio vs. Bom \\
\hline \multirow{2}{*}{ Médicos/Enfermeiros } & 2.58 vs. 3.14 & 2.58 vs. 3.43 & 3.14 vs. 3.43 \\
\hline & $(p=0.087)$ & $(p=0.003) *$ & $(p=0.121)$ \\
\hline \multirow{2}{*}{ Revistas/Jornais/TV } & 2.54 vs. 2.47 & 2.54 vs. 2.46 & 2.47 vs. 2.46 \\
\hline & $(\mathrm{p}=0.939)$ & $(p=0.903)$ & $(p=0.987)$ \\
\hline \multirow{2}{*}{ Amigos/familiares } & 2.54 vs. 2.35 & 2.54 vs. 2.15 & 2.35 vs. 2.15 \\
\hline & $(p=0.693)$ & $(p=0.202)$ & $(p=0.267)$ \\
\hline \multirow{2}{*}{ Formação profissional } & 2.58 vs. 3.20 & 2.58 vs. 3.20 & 3.20 vs. 3.20 \\
\hline & $(p=0.018)^{*}$ & $(p=0.017)^{*}$ & $(p=1.000)$ \\
\hline Colegas profissionais de desporto & $\begin{array}{c}2.58 \text { vs. } 2.58 \\
(p=1.000)\end{array}$ & $\begin{array}{c}2.58 \text { vs. } 2.68 \\
(p=0.872)\end{array}$ & $\begin{array}{c}2.58 \text { vs. } 2.68 \\
(p=0.654)\end{array}$ \\
\hline Colegas professores & $\begin{array}{c}2.37 \text { vs. } 2.26 \\
(p=0.844)\end{array}$ & $\begin{array}{c}2.37 \text { vs. } 2.51 \\
(p=0.784)\end{array}$ & $\begin{array}{l}2.26 \text { vs. } 2.51 \\
\quad(p=0.082)\end{array}$ \\
\hline Redes sociais na internet & $\begin{array}{c}1.75 \text { vs. } 1.70 \\
(p=0.959)\end{array}$ & $\begin{array}{c}1.75 \text { vs. } 1.33 \\
(p=0.049)^{*}\end{array}$ & $\begin{array}{c}1.70 \text { vs. } 1.33 \\
(p=0.001)^{*}\end{array}$ \\
\hline
\end{tabular}

\section{DISCUSSÃO}

A investigação feita centrou-se na avaliação do conhecimento dos professores de Educação Física portugueses para atuar em situações de emergência e na possível influência que fatores como idade, género, habilitações literárias/formação académica, perceção do conhecimento ou fontes de informação têm sobre esse conhecimento.

O professor de Educação Física é frequentemente o primeiro agente a socorrer uma vítima de acidente escolar, quer no contexto de aula quer nas atividades de desporto escolar, e a sua avaliação e capacidade de atuar têm importantes consequências na recuperação dos alunos acidentados (Flegel, 2002; Olympia et al., 2005; Patsaki et al., 2012). Um nível baixo de conhecimento sobre como atuar em situações de emergência pode condicionar a recuperação da vítima, por não lhes terem sido prestados os cuidados de $1^{\text {os }}$ socorros mais adequados (Başer et al., 2007; Wang et al., 2012).

Os resultados obtidos indicam que apenas $19.0 \%$ dos professores entrevistados tem um bom nível de KT relativamente aos modos de atuação em situações de emergência, sendo que $58.1 \%$ apresentam um nível médio e $22.9 \%$ um nível mau de conhecimento. Ao nível do KO,
49.5\% dos professores analisados tem um bom nível de conhecimento operacional comparativamente aos modos atuação em situações de emergência, sendo que $41.6 \%$ apresentam um nível médio e apenas $9.0 \%$ um nível mau de conhecimento operacional. Embora o KO apresente melhores resultados que o $\mathrm{KT}$, ainda há metade dos professores com nível médio ou mau, revelando um deficit de conhecimento sobre como atuar em situações de emergência. Os resultados obtidos são parcialmente concordantes com os apresentados por Olympia et al. (2005), Emery e Tyreman (2009), Wang et al. (2012), que referem um mau conhecimento dos professores de Educação Física sobre como lidar com situações de emergência, particularmente ao nível do KT. No entanto, ao nível do $\mathrm{KO}$, cerca de metade dos professores apresenta um bom nível de conhecimento, o que vai de encontro ao referido por Patsaki et al. (2012) e Abraldes e Ortín (2010). Como síntese dos resultados obtidos da avaliação do conhecimento dos professores para atuar em situações de emergência pode considerar-se que o conhecimento sobre como atuar face a um acidente (KO) é médio, apesar dos conhecimentos teóricos sobre $1^{\text {os }}$ socorros serem consideravelmente piores. 
Considerando os resultados obtidos, é fundamental que o docente desenvolva conhecimento de como agir (KO), para o bem-estar do jovem acidentado. Quanto mais conhecimento o Professor possuir maior será a capacidade de atuar (Abernethy et al., 2003; Başer et al., 2007; Fioruc et al., 2008; Wang et al., 2012).

Quanto aos fatores que influenciam quer o KT quer o KO, tem-se que a idade condiciona quer o KT quer o KO: os professores mais novos têm um melhor conhecimento para lidar com situações de emergência e os professores com idades superiores a 50 anos um pior conhecimento para reagir face à emergência.

Os resultados obtidos reportam que o género não é um fator com influência no KT e no KO. Estes resultados corroboram os apresentados por Ortín e Abraldes (2007), em que os autores consideram não haver diferença no conhecimento para agir face a uma emergência entre professoras e professores.

Quanto às habilitações literárias (considerando apenas licenciados e mestres, por o número de doutorados não ser estatisticamente significativo) têm-se que os mestres apresentam um melhor nível de KT, mas um igual nível de KO, isto é, as habilitações literárias não alteram o conhecimento "prático" que os professores têm sobre como lidar com situações de emergência. Este resultado difere parcialmente das conclusões de Abraldes, Córcoles, Muñoz, e Moreno (2011), num estudo para avaliar o nível de conhecimentos de $1^{\text {os }}$ socorros de estudantes universitários de Atividade Física e Desporto da Universidade do Porto, em que os autores consideram que o nível de conhecimento dos estudantes finalistas é baixo por estes conteúdos se abordarem principalmente no $2^{\circ}$ ciclo de estudos (Mestrado). Para os autores, os mestres teriam um melhor nível de conhecimentos sobre $1^{\text {os }}$ socorros, resultado que a presente investigação apenas corrobora relativamente ao KT.

No que respeita à perceção que os professores têm do próprio conhecimento, tem-se que os professores com nível "Mau" de KT consideram que não estão tão bem informados sobre como reagir a uma situação de emergência. Os docentes com nível "Mau" de KT e de KO percecionam ter significativamente mais dúvidas sobre o que fazer em caso de emergência relativamente aos outros docentes com níveis "Bom" e "Médio". Estes resultados sugerem que os professores inquiridos têm uma boa perceção do seu próprio conhecimento e das suas limitações para lidarem com situações de emergência. Esta observação vai de encontro ao referido por Abraldes e Ortín (2010), i.e., os professores têm a consciência da necessidade de encontrar novos conhecimentos para lidar com situações de emergência.

Finalmente o último fator investigado foram as fontes de informação usadas pelos professores para obterem conhecimento sobre como lidar com situações de emergência. As principais fontes reportadas pelos professores são médicos/enfermeiros, formação profissional e a licenciatura. Apesar de serem escassos os estudos que mostrem quais as fontes de informação sobre $1^{\text {os }}$ socorros usadas pelos professores, Levenson, Morrow, Morgan, e Pfefferbaum (1986) apresentam resultados semelhantes, indicando que o conhecimento dos professores sobre conceitos de saúde provém maioritariamente de profissionais de saúde e formação universitária e profissional.

Pelos resultados obtidos na nossa investigação, os docentes com nível "Bom" de KT recorrem significativamente mais à consulta de media como Jornais/Revistas/TV do que os docentes com nível "Médio" $(p=0.000)$ e "Mau" $(p=0.001)$. Este facto pode estar relacionado com a melhoria de KT, uma vez que segundo Eysenbach (2008) quanto maior o conhecimento do utilizador melhor a sua capacidade de filtrar a informação pertinente dos media. Para este resultado pode ainda contribuir o facto das informações disseminadas por esses media serem alvo de revisão editorial (Lai \& Wong, 2002).

Os resultados obtidos permitem concluir ainda que os docentes com nível "Mau" de KO utilizam significativamente menos a "Formação profissional" do que os outros docentes. Estes 
resultados são parcialmente suportados pela investigação de Abraldes e Ortin (2010), em que os autores notam uma tendência para melhor conhecimento sobre $1^{\text {os }}$ socorros em professores que tiveram ações de formação específica, apesar de não terem diferenças significativas entre os grupos.

Importa também referir que as redes sociais e amigos/família são de forma geral pouco consultados pelos docentes para saber o que fazer em caso de emergência. Porém observamos que os docentes com nível "Bom" de KO procuram significativamente menos do que os docentes com nível "Médio" $(p=0.001)$ e "Mau" $(p=0.049)$. A qualidade de informação existente nas redes sociais, onde há muitos mitos e não-verdades sobre como lidar com acidentes (Oh, Lauckner, Boehmer, Fewins-Bliss, \& Li, 2013) pode ser uma das razões para que este meio não seja privilegiado, especialmente pelo grupo com nível de conhecimento "Bom".

Esta investigação apresenta, como principais limitações, a relativa baixa taxa de resposta dos professores de EF (a amostra corresponde a apenas $4.4 \%$ dos professores de EF em Portugal) e a falta de conhecimento do vínculo laboral dos professores entrevistados, o que pode condicionar o tipo de resposta dada pelos docentes. Outra limitação é o facto do conhecimento operacional ter sido avaliado por questionário e não por análise concreta da resposta dos professores à situação de emergência, por exemplo, com situações práticas simuladas. Neste estudo também não foi feita uma análise do tipo de formação superior dos professores (curso/universidade) nem dos conteúdos programáticos dos cursos frequentados, o que limitou as conclusões relativamente à influência das habilitações literárias dos docentes no nível de conhecimento.

\section{CONCLUSÕES}

Nesta investigação procurámos avaliar o conhecimento teórico (KT) e o conhecimento operacional (KO) dos professores de Educação Físicas portugueses. Ao contrário do que acontece com outros países, em Portugal são escassos os dados sobre a caracterização desta população quanto à capacidade de atuar em acidente escolar. A presente investigação reveste-se, por isso, de um carácter inovador pela caracterização realizada e ainda pela avaliação da influência de alguns fatores (idade, género, habilitações literárias/formação académica, perceção do conhecimento e fontes de informação sobre conhecimento de $1^{\text {os }}$ socorro) nessa atuação.

Concluímos que os professores apresentam um nível de KT inferior ao de KO, o que significa que os professores têm mais conhecimentos operacionais que teóricos. Por outro lado, mais de metade dos professores têm um nível "Mau "ou "Médio" de KO, pelo que ainda há bastante trabalho a fazer de modo a melhorar a resposta que o professor dá, como $1^{\circ}$ agente e responsável pela prestação de $1^{\text {os }}$ socorros. Este conhecimento dos professores de EF difere com a idade, sendo significativamente melhor nos professores mais jovens. Não é alterado pelo género, mas os resultados mostram que os docentes mestres apresentam um melhor nível de KT, apesar de não haver diferenças significativas no KO. Os professores mostram uma boa perceção do seu nível de conhecimento para lidar com situações de emergência em contexto escolar. A informação que os professores têm sobre como reagir face a uma emergência provém principalmente de médicos/enfermeiros, formação profissional e licenciatura, sendo pouco procurada nas redes sociais e nos amigos/família. Concluímos porem que os docentes com nível "Bom" de KT utilizam significativamente mais os jornais/revistas/TV como fonte de informação, relativamente aos outros docentes. Por sua vez os docentes com nível "Mau" de KO procuram significativamente menos a formação profissional como fonte informação, quando comparados com os outros docentes.

Assim é fundamental promover a formação contínua dos professores de Educação Física na área dos $1^{\text {os }}$ socorros. Este conhecimento pode ser adquirido com a inclusão de módulos de $1^{\text {os }}$ socorros na formação académica (licenciatura ou mais especificamente no mestrado via ensino), ou com a utilização de novas tecnologias 
através da construção de plataformas e-learning ou os MOOC (Massive Open Online Courses), cada vez mais utilizadas pelas pessoas com o objetivo de aprender e adquirir novas experiências e pelas instituições como meio de disseminação de conhecimento. Não esquecer que na definição e construção dos conteúdos, o uso de formadores que sejam médicos/enfermeiros, no contexto da formação profissional, poderá ser uma mais-valia para a formação dos professores neste âmbito.

\section{Agradecimentos:}

Agradecemos a colaboração do Mestre Ricardo Oliveira, responsável pela recolha de dados e a todos os professores de EF que aceitaram participar neste estudo.

Conflito de Interesses:

Nada a declarar.

Financiamento:

Nada a declarar.

\section{REFERÊNCIAS}

Abernethy, L., MacAuley, D., McNally, O., \& McCann, S. (2003). Immediate care of school sport injury. Injury Prevention, 9(3), 270-273. http://doi.org/10.1136/ip.9.3.270

Abraldes, J. A., Córcoles, C. M., Muñoz, C. M., \& Moreno, A. (2011). Valoración de los primeros auxilios en estudiantes de Educación Física. Trances, 3(1), 88-104.

Abraldes, J. A., \& Ortín, A. (2010). Conocimiento en primeros auxilios de los profesores de Educación Física en ESO. Revista Internacional de Medicina y Ciencias de la Actividad Física y del Deporte, 10(38), 271-283.

Başer, M., Coban, S., Taşci, S., Sungur, G., \& Bayat, M. (2007). Evaluating first-aid knowledge and attitudes of a sample of Turkish primary school teachers. Journal of Emergency Nursing: JEN: Official Publication of the Emergency Department Nurses Association, 33(5), 428-432. http://doi.org/10.1016/j.jen.2006.11.003

Bouchard, C., Blair, S. N., \& Haskell, W. (Eds.). (2012). Physical Activity and Health-2nd Edition (2. ${ }^{a}$ ed.). Human Kinetics.
Emery, C., \& Tyreman, H. (2009). Sport participation, sport injury, risk factors and sport safety practices in Calgary and area junior high schools. Paediatrics \& Child Health, 14(7), 439-444.

Eysenbach. (2008). Credibility of Health Information and Digital Media: New Perspectives and Implications for Youth. Em M. J. Metzger \& A. J. Flanagin (Eds.), Digital Media, Youth, and Credibility (The John D. and Catherine T. MacArthur Foundation Series on Digital Media and Learning, pp. 123-154). Cambridge, MA: The MIT Press. Obtido de https://tspace.library.utoronto.ca/bitstream/1807/9906/6/Eysenbach2007.pdf

Fioruc, B. E., Molina, A. C., Junior, W. V., \& Lima, S. M. (2008). Educação em saúde: abordando primeiros socorros em escolas públicas no interior de São Paulo. Revista Eletrônica de Enfermagem, 10(3), 695-702.

Flegel, M. (2002). Primeiros socorros no esporte. São Paulo: Manole.

Junkins, E. P., Knight, S., Lightfoot, A. C., Cazier, C. F., Dean, J. M., \& Corneli, H. M. (1999). Epidemiology of School Injuries in Utah: A Population-Based Study. Journal of School Health, 69(10), 409-412. http://doi.org/10.1111/j.17461561.1999.tb06360.x

Lai, G., \& Wong, O. (2002). The tie effect on information dissemination: the spread of a commercial rumor in Hong Kong. Social Networks, 24(1), 49-75. http://doi.org/10.1016/S03788733(01)00050-8

Levenson, P. M., Morrow, J. R., Morgan, W. C., \& Pfefferbaum, B. J. (1986). Health Information Sources and Preferences as Perceived by Adolescents, Pediatricians, Teachers and School Nurses. The Journal of Early Adolescence, 6(2), 183-195. http://doi.org/10.1177/0272431686062008

Liberal, E. F., Aires, R. T., Aires, M. T., \& Osório, A. C. de A. (2005). Safe school. Jornal de Pediatria, 81(5), s155-s163. http://doi.org/10.1590/S002175572005000700005

Linakis, J. G., Amanullah, S., \& Mello, M. J. (2006). Emergency department visits for injury in school-aged children in the United States: a comparison of nonfatal injuries occurring within and outside of the school environment. Academic Emergency Medicine, 13(5), 567570.

http://doi.org/10.1197/j.aem.2005.11.073

Miller, T. R., \& Spicer, R. S. (1998). How safe are our schools? American Journal of Public Health, $88(3)$, 413-418.

http://doi.org/10.2105/AJPH.88.3.413 
Oh, H. J., Lauckner, C., Boehmer, J., Fewins-Bliss, R., \& Li, K. (2013). Facebooking for health: An examination into the solicitation and effects of health-related social support on social networking sites. Computers in Human Behavior, 29(5), 2072-2080. http://doi.org/10.1016/j.chb.2013.04.017

Olympia, R. P., Wan, E., \& Avner, J. R. (2005). The preparedness of schools to respond to emergencies in children: a national survey of school nurses. Pediatrics, 116(6), e738-745. http://doi.org/10.1542/peds.2005-1474

Orchard, J. W., \& Finch, C. F. (2002). Australia needs to follow New Zealand's lead on sports injuries. The Medical Journal of Australia, 177(1), 3839.

Ortín, A., \& Abraldes, J. A. (2007). Knowledge of first aids in the physical education teachers of secondary obligatory education. An analysis of gender role. Em J. A. Abraldes \& N. Rodríguez (Eds.), Book of abstracts International Lifesaving Congress 2007 (p. 128). La Coruña: Federación de Salvamento e Socorrismo de Galicia (FESSGA). Obtido de http://fessga.es/Documentos/Publicaciones/00417.pdf

Patsaki, A., Pantazopoulos, I., Dontas, I., Passali, C., Papadimitriou, L., \& Xanthos, T. (2012). Evaluation of Greek high school teachers' knowledge in basic life support, automated external defibrillation, and foreign body airway obstruction: implications for nursing interventions. Journal of Emergency Nursing: JEN: Official Publication of the Emergency Department Nurses Association, 38(2), 176-181. http://doi.org/10.1016/j.jen.2010.09.002

Pinheiro, P., Esteves, D., \& Brás, R. (2011). Evaluation of new information technologies exposure on knowledge retention regarding benefits of physical activity on health status. The Electronic Journal Information Systems Evaluation, 14(1), 122-133.

Prédine, R., Chau, N., Lorentz, N., Prédine, E., Legras, B., Benamghar, L., ... Mergel, B. (2002). Les accidents scolaires dans des établissements d'enseignement général: incidence, causes, et conséquences. Revue D'épidémiologie Et De Santé Publique, 50(3), 265-276.

Ransone, J., \& Dunn-Bennett, L. R. (1999). Assessment of first-aid knowledge and decision making of high school athletic coaches. Journal of Athletic Training, 34(3), 267-271.

Reis, M. M. S. (2005). Acidentes escolares nos agrupamentos de escolas de Braga entre 1998 e 2003 (Dissertação de Mestrado em Estudos da Criança - Promoção da Saúde e Meio Ambiente). Universidade do Minho, Braga.

Strong, W. B., Malina, R. M., Blimkie, C. J. R., Daniels, S. R., Dishman, R. K., Gutin, B., ... Trudeau, F. (2005). Evidence based physical activity for school-age youth. The Journal of Pediatrics, 146(6),

732-737. http://doi.org/10.1016/j.jpeds.2005.01.055

Wang, C. K. J., \& Koh, T. H. M. (2006). Sport ability beliefs, self-determination and beliefs about the purposes of physical education among Singaporean potential preservice teachers. Asian Journal of Exercise and Sports Science, 3(1), 25-34.

Wang, K.-M., Lin, Y.-H., \& Huang, Y.-C. (2012). The Knowledge and Attitude of Sports Injury Prevention and Management of Senior High School Athletes in Taiwan. International Journal of Sport and Health Science, 10, 12-22. http://doi.org/10.5432/ijshs.201122

World Health Organization. (2010). Global recommendations on physical activity for health. Genebra: World Health Organization. Obtido de http://whqlibdoc.who.int/publications/2010/9789241599979_eng.pdf

Todo o conteúdo da revista Motricidade está licenciado sob a Creative Commons, exceto quando especificado em contrário e nos conteúdos retirados de outras fontes bibliográficas. 